\title{
Primary apocrine adenocarcinoma of the orbit with lacrimal sac invasion
}

\author{
Mona Mohammad $\mathbb{D}^{1}$, Rashed Mustafa Nazzal $\mathbb{\circledR}^{5}$, Maysa AlHussaini $\mathbb{(}^{2}$, Niveen Abdullah $\mathbb{(}^{2}$, \\ Alaa Saleh $\mathbb{B}^{3}$, Sana' Muhsen $\mathbb{1}^{4}$, Robert Rejdak $\mathbb{1}^{6}$, Yacoub A. Yousef $\left(\mathbb{C}^{1}\right.$ \\ ${ }^{1}$ Department of Surgery/Ophthalmology, King Hussein Cancer Center (KHCC), Amman, Jordan \\ ${ }^{2}$ Department of Pathology, King Hussein Cancer Center (KHCC), Amman, Jordan \\ ${ }^{3}$ Department of and Radiology, King Hussein Cancer Center (KHCC), Amman, Jordan \\ ${ }^{4}$ Ophthalmology Department, Jordan University Hospital, University of Jordan, Amman, Jordan \\ ${ }^{5}$ Shami Eye Center, Amman, Jordan \\ ${ }^{6}$ Medical University of Lublin, Lublin, Poland
}

\begin{abstract}
BACKGROUND: Orbital adenocarcinoma usually is metastatic and rarely can be primary disease of the orbit with no clear standard of care. Herein, we report a case of primary apocrine adenocarcinoma in the orbit with invasion into lacrimal sac in a young patient.

CASE REPORT: A 38-year-old male presented with enlarging lump in the left eye. Ocular imaging studies showed an orbital mass with invasion into the lacrimal sac and surrounding muscles. The lesion was found to be adenocarcinoma with apocrine differentiation in both pathology and immunohistochemistry. Full body imaging studies showed no extra ocular spread and no other tumors to suggest orbital metastasis. The patient was treated with orbital exenteration and was given adjuvant local radiotherapy and systemic chemotherapy because of positive margins and high risk pathological features. On serial exams over 5 years follow up, the patient was free of local recurrence or distant metastasis.

CONCLUSION: Orbital adenocarcinoma can present as a primary orbital tumor in young adults originating from apocrine glands, and aggressive surgical resection can be lifesaving management.
\end{abstract}

KEY WORDS: adenocarcinoma; apocrine; metastasis; orbit

Ophthalmol J 2020; Vol. 5, 38-41

\section{INTRODUCTION}

There are two types of sweat glands: apocrine and eccrine. Unlike eccrine glands, apocrine glands are distributed in limited areas in the body including the axilla, anogenital areas, mammary glands, and eyelids [1]. Characteristically, these glands secrete by decapitation; part of the cytoplasm of the glandular cell is pushed off into the glandular lumen [2]. The glands of Moll are modified apocrine glands that lie in the base of the follicles of the eyelashes [3].
Adenocarcinoma (AC) is a rare, slow-growing skin carcinoma with apocrine differentiation arising from apocrine glands. This is rarely seen in the orbit, but common in the axilla and the anogenital region [4]. Adenocarcinoma in the orbit is usually metastasis from breast or lung adenocarcinoma, but can be rarely primary in the ocular region, where they are usually derived from Moll glands of the eyelid [5]. Adenocarcinoma should be recognized and treated properly because of a high rate of both local recurrence and distant metastasis [6]. Herein 
we are reporting an extremely rare case of primary orbital adenocarcinoma in a young patient who was cured by orbital exenteration.

\section{CASE REPORT}

A 38-year-old previously healthy male patient referred to the ophthalmology department at King Hussein Cancer Centre (KHCC) with a slowly-enlarging, painless lump in the medial corner of the left eye of four months duration. On clinical examination, best corrected visual acuity was 20/20 in both eyes. A mass overlying the left caruncle was grossly visible. It was not attached to eyelids. Motility testing revealed a limitation of abduction in the left eye. This was associated with binocular diplopia on left gaze. Hertel exophthalmometry showed no proptosis. Slit-lamp examination of both the anterior and posterior segments was normal. Examination of the right eye was unremarkable. Intraocular pressure was normal in both eyes. Palpation of regional lymph nodes showed no palpable preauricular, submandibular or cervical nodes.

Orbital magnetic resonance imaging (MRI) with contrast was requested. It showed an intensely enhancing mass involving the anterior aspect of the left orbit medially. It measured $2.3 \times 1.1$ centimeters $(\mathrm{cm})$ in the axial plane. The mass was invading the lacrimal sac and the upper part of the left nasolacrimal duct. It abutted the anterior eye globe, medial rectus, and inferior oblique muscles. The mass was inseparable from the overlying skin but was not arising from the skin (Fig. 1). Orbital computed tomography CT scan with contrast showed focal destruction of the lacrimal bone (Fig. 2).

Systemic staging including a whole body CT scan did not reveal any tumors elsewhere. A bone scan was done and it showed no convincing evidence of osteoblastic metastases. No tumorous lesions suggestive of primary apocrine adenocarcinoma were found elsewhere in the skin, particularly in axillae or around the nipples. Incisional biopsy for the left orbital mass was undertaken. The pathology examination revealed a moderately differentiated apocrine adenocarcinoma (Fig. 3).

On immunohistochemistry, tumor cells were positive for androgen receptor (AR), cytokeratin 7 (CK7), cytokeratin 19 (CK19), cytokeratin 18 (CK18), epithelial membrane antigen (EMA), polyclonal carcinoembryonic antigen (CEA), gross cystic disease fluid protein 15 (GCDFP-15), CD99 and MUC1 immunostains. They were
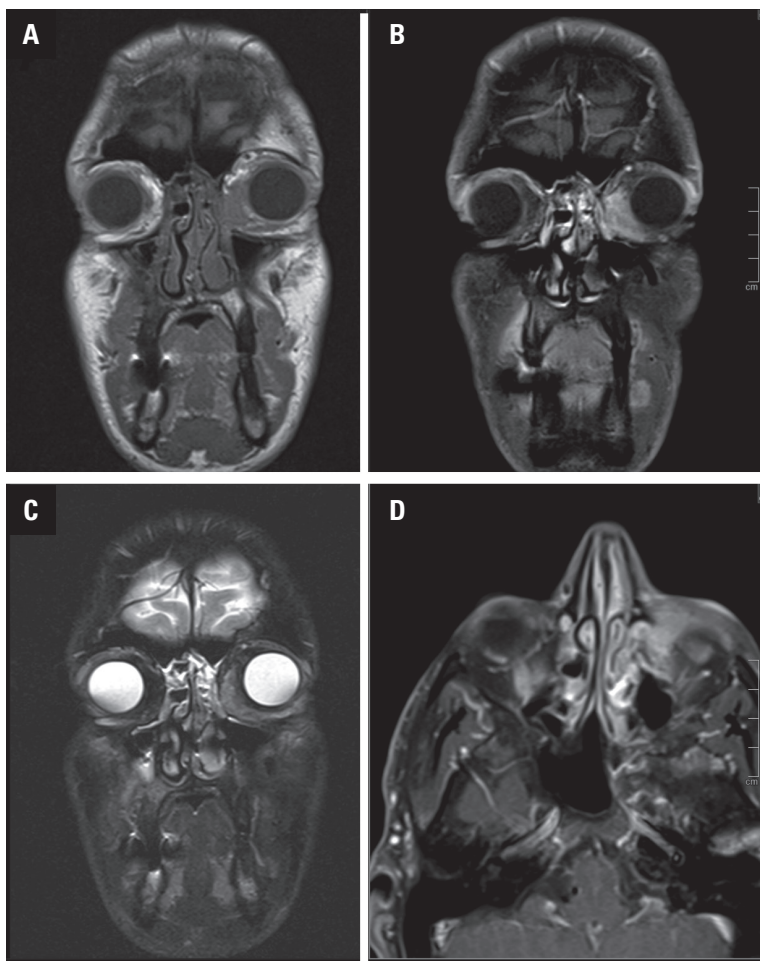

FIGURE 1. A. Orbit MRI coronal view T1; B. T1 post contrast fat sat; C. Short TI inversion recovery (STIR); D. Axial T1 post contrast fat sat. A lobulated mass at left medial canthus invading the lacrimal sac and the medial orbital rim. The mass is inseparable from anterior eye globe, medial rectus and inferior oblique muscles. The mass is invading the lacrimal sac and the upper part of the left nasolacrimal duct
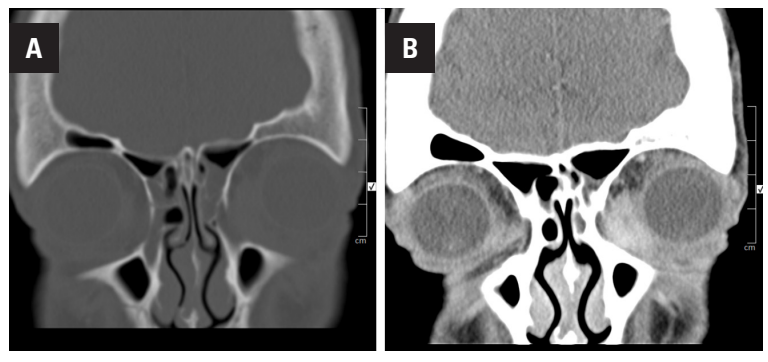

FIGURE 2. A. Orbit CT with contrast coronal views bone; B. Soft tissue (B) windows. A lobulated soft tissue mass (arrow) at left medial canthus, invading the left lacrimal sac $(\mathbf{A})$ with focal destruction of the lacrimal bone (B)

negative for cytokeratin 20 (CK-20), monoclonal carcinoembryonic antigen (CEA), CDX-2, prostate-specific antigen (PSA), renal cell carcinoma (RCC), PAX8, vimentin, thyroid transcription factor-1 (TTF-1), hepatocyte specific antigen (Hep Par-1), Sal-like protein 4 (SALL4), MUC2, MUC5, thyroglobulin, CA19-9, S100 protein, CD34, surfactant, estrogen receptor (ER), and factor VIII-re- 


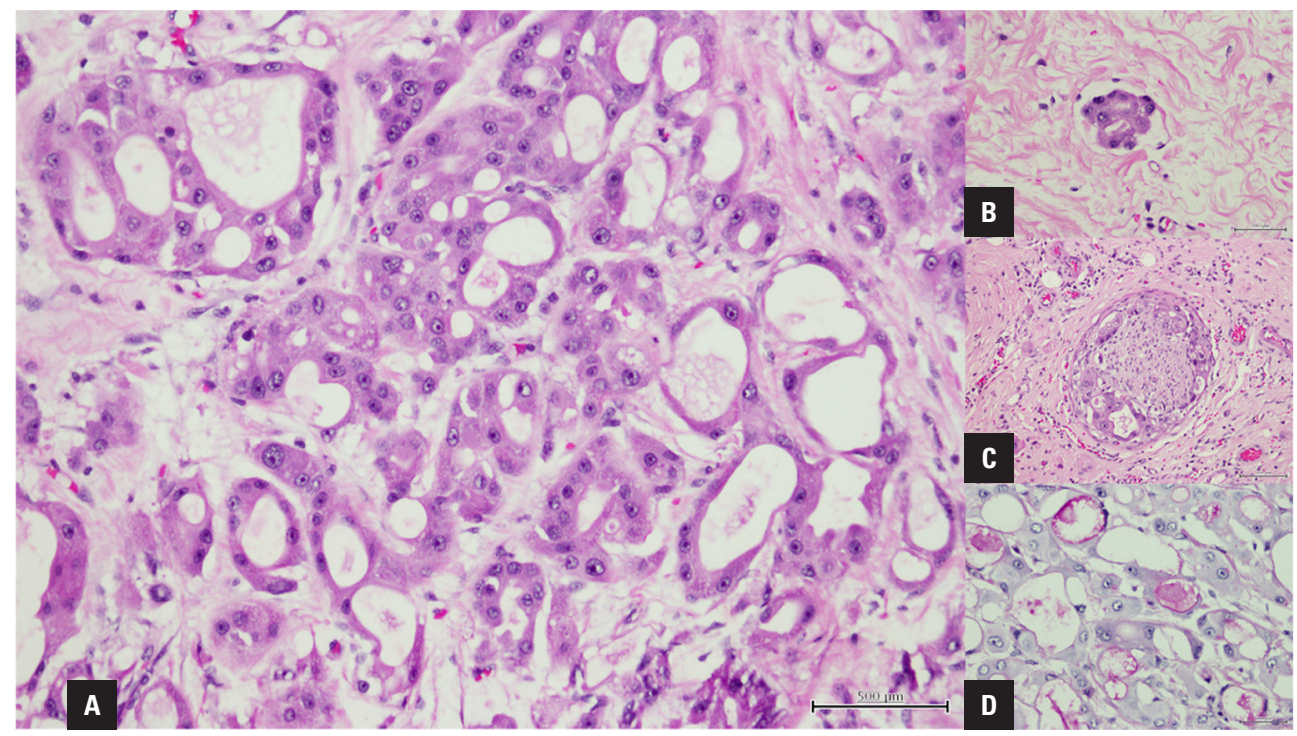

FIGURE 3. A. The tumor is composed of well-formed glands, with an irregular lumen and pseudopapillary structures lined by cells with abundant acidophilic granular cytoplasm, large vesicular nuclei with single prominent nucleoli. Secretion decapitation at the apical border of the tumor cells is seen focally. The lumens contain secretions. In addition, few cells show intracytoplasmic vacuoles containing mucin droplets, (H\&E X20). B. Tumor lymphatic permeation (H\&E X40). C. Perineural invasion (H\&E X40). D. PAS/PASD special stain highlighted the mucin within the glands and in the cytoplasm of some of the cells (PAS/PASD stain, X40)

lated antigen. These results ruled out systemic metastasis to the orbit or other primary tumors in the breast or skin.

Based on all of the above findings, the final diagnosis was left orbital primary apocrine adenocarcinoma. Therefore, we proceeded with left eyelid-sparing orbital exenteration. Histopathology of the left orbit showed moderately differentiated apocrine adenocarcinoma with positive margins and lymphovascular invasion (pT3bN0). Given these high-risk pathological features, the patient was given adjuvant chemoradiotherapy; 66 Gray (Gy) in 33 fractions to the left socket and intravenous Cisplatin.

Follow up with serial orbit and neck MRI scans, showed no local recurrence or regional lymph nodes enlargement. The patient has been disease-free for the last 5 years with no signs of local or systemic recurrence of the tumor.

\section{DISCUSSION}

Apocrine adenocarcinoma (AC) is a rare, slow-growing skin carcinoma with apocrine differentiation arising from apocrine glands. AC occurs most commonly in the axilla followed by the anogenital region [4]. These tumors may arise rarely in the ocular region, where they are usually derived from Moll glands of the eyelid [5]. AC should be recognized and treated properly because of a high rate of both local recurrence and distant metastasis [6].

Kipkie and Haust described the following criteria for the histologic diagnosis of apocrine adenocarcinoma: Periodic acid-Schiff (PAS) positive, strongly eosinophilic cytoplasm, decapitation secretion, iron-positive intracellular pigment, and occurrence in areas where apocrine glands are normally located. The PAS-positive malignant apocrine epithelial cells often have diastase-resistant granules containing iron [7].

Immunohistochemistry has a role in diagnosing these tumors, particularly in poorly differentiated specimens. GCDFP-15 as a specific tissue marker of apocrine epithelium was found to stain apocrine-related neoplasms in $84 \%$ of the specimens [8].

Apocrine adenocarcinoma may represent a primary skin tumor, or be secondary to various internal adenocarcinomas that metastasize to the skin [9]. In a major review of 19 cases of AC of the eyelid published by Figueira et al., fifty-three percent of cases had tumors limited to the eyelids. Most patients (18 out of 19) presented with a painless blue-brown eyelid lump. Local orbital and periorbital invasion was described in $47 \%$ of the patients [10]. In a case reported by Hoang et al., apocrine adenocarcinoma in the lacrimal sac presented as a masquerading chronic dacryocystitis [11]. In another case reported 
by Shintaku et al., the neoplasm was located in the subcutaneous tissue of the eyelid in the medial canthus and showed a subconjunctival spread in the lacrimal caruncle [12].

In this case report, the patient had no eyelid skin mass as is the usual case for these tumors. Instead, the tumor occurred primarily in the caruncle with local invasion to the lacrimal sac. Given the fact that there are no apocrine glands in the lacrimal sac region [13], we think that the tumor probably originated in the Moll's gland of the eyelid near the region of the medial canthus and subsequently exhibited a subconjunctival spread into the lacrimal caruncle instead of moving anteriorly toward the eyelid skin. This is similar to the case published by Shintaku et al. [12] The other possibility is that the tumor could have arisen from embryonic epithelial remnants within the orbit.

The possibility of a secondary tumor was excluded by the normal results of the systemic workup and immunohistochemistry. This is consistent with the final diagnosis of the presented case as a primary apocrine adenocarcinoma of the caruncle with lacrimal sac invasion.

\section{Acknowledgments/Disclosure}

None.

\section{REFERENCES}

1. Weedon D. Skin Pathology. Churchill Livingstone, Sydney 2002: 877888.

2. Gesase AP, Satoh Y. Apocrine secretory mechanism: recent findings and unresolved problems. Histol Histopathol. 2003; 18(2): 597-608, doi: 10.14670/HH-18.597, indexed in Pubmed: 12647810.
3. Stoeckelhuber M, Stoeckelhuber BM, Welsch U. Human glands of Moll: histochemical and ultrastructural characterization of the glands of Moll in the human eyelid. J Invest Dermatol. 2003; 121(1): 28-36, doi: 10.1046/.1523-1747.2003.12328.x, indexed in Pubmed: 12839560.

4. Paties C, Taccagni GL, Papotti M, et al. Apocrine carcinoma of the skin. A clinicopathologic, immunocytochemical, and ultrastructural study. Cancer. 1993; 71(2): 375-381, doi: 10.1002/1097-0142(19930115)71:2<375::aidcncr2820710218>3.0.c0;2-4, indexed in Pubmed: 7678545.

5. Barker-Griffith AE, Streeten BW, Charles NC. Moll gland neoplasms of the eyelid: a clinical and pathological spectrum in 5 cases. Arch Ophthalmol. 2006; 124(11): 1645-1649, doi: 10.1001/ archopht.124.11.1645, indexed in Pubmed: 17102015.

6. Warkel RL, Helwig EB. Apocrine gland adenoma and adenocarcinoma of the axilla. Arch Dermatol. 1978; 114(2): 198-203, indexed in Pubmed: 629545

7. Kipkie GF, Haust MD. Carcinoma of apocrine glands; report of case. AMA Arch Derm. 1958; 78(4): 440-445, doi: 10.1001/ archderm.1958.01560100014003, indexed in Pubmed: 13582185.

8. Mazoujian G, Pinkus GS, Davis S, et al. Immunohistochemistry of a gross cystic disease fluid protein (GCDFP-15) of the breast. A marker of apocrine epithelium and breast carcinomas with apocrine features. Am J Pathol. 1983; 110(2): 105-112, indexed in Pubmed: 6130702.

9. Hood Cl, Font RL, Zimmerman LE. Metastatic mammary carcinoma in the eyelid with histiocytoid appearance. Cancer. 1973; 31(4): 793-800, doi: 10.1002/1097-0142(197304)31:4<793::aidcncr2820310406 > 3.0.c0;2-a, indexed in Pubmed: 4350331.

10. Figueira EC, Danks J, Watanabe A, et al. Apocrine adenocarcinoma of the eyelid: case series and review. Ophthalmic Plast Reconstr Surg. 2013; 29(6): 417-423, doi: 10.1097/IOP.0b013e3182a64fe5, indexed in Pubmed: 24217473.

11. Hoang A. Apocrine Adenocarcinoma of Lacrimal Sac Presenting as Chronic Dacryocystitis. J Ophthal Clin Res. 2017; 4(1): 1-2, doi: 10.24966/ocr-8887/100027.

12. Shintaku M, Tsuta K, Yoshida H, et al. Apocrine adenocarcinoma of the eyelid with aggressive biological behavior: report of a case. Pathol Int. 2002; 52(2): 169-173, doi: 10.1046/j.1440-1827.2002.01323.x, indexed in Pubmed: 11940224.

13. Kominami R, Yasutaka $S$, Taniguchi $Y$, et al. Anatomy and histology of the lacrimal fluid drainage system. Okajimas Folia Anat Jpn. 2000; 77(5): 155-160, doi: 10.2535/ofaj1936.77.5_155, indexed in Pubmed: 11218713. 